\title{
EVOLUÇÃO TEÓRICA SOBRE CAPACIDADES DINÂMICAS: ANÁLISES E PROPOSIÇÓES POR MEIO DO ESTUDO BIBLIOMÉTRICO NACIONAL
}

\author{
THEORETICAL EVOLUTION ON DYNAMIC CAPACITIES: ANALYSIS AND PROPOSITIONS \\ THROUGH THE NATIONAL BIBLIOMETRIC STUDY
}

\author{
Felipe Zaluski
}

Universidade Federal de Santa Maria, Santa Maria, RS, Brasil, felipezaluski@hotmail.com

\section{Ana Paula da Rosa Dezordi}

Universidade Regional do Noroeste do Estado do Rio Grande do Sul, Ijuí, RS, Brasil, annna.darosa@gmail.com

\section{Jorge Oneide Sausen (iD}

Universidade Regional do Noroeste do Estado do Rio Grande do Sul, Ijuí, RS, Brasil, josausen@unijui.edu.br

Glória Charão Ferreira

Universidade Regional do Noroeste do Estado do Rio Grande do Sul, Ijuí, RS, Brasil, gcfconsultoria@gmail.com

\section{Clandia Maffini Gomes}

Universidade Federal de Santa Maria, Santa Maria, RS, Brasil, clandiamg@gmail.com

Resumo: O objetivo deste estudo é debater a evolução da produção científica brasileira sobre a temática das Capacidades Dinâmicas por meio de uma revisão sistemática da literatura na base Scientific Periodicals Eletronic Library - SPELL (2003-2019). Primeiramente, destaca-se as origens conceituais, divergências e convergências teóricas. Em seguimento, analisa-se a evolução da produção teórica científica. Por fim, efetua-se uma análise das temáticas e sugestôes de estudos futuros dos artigos. Conclui-se que houve um crescente avanço teórico nas duas últimas décadas e que pode avançar nos próximos anos. Destaca-se as contribuiçóes da agenda de pesquisa e compreensão de como os estudos sobre a temática vem sendo desenvolvidos no âmbito nacional brasileiro.

Palavras-chave: Capacidades Dinâmicas. Evolução Teórica. Produção Nacional Brasileira.

\begin{abstract}
The objective of this study is to discuss the evolution of Brazilian scientific production on the theme of Dynamic Capabilities through a systematic review of the literature in the Scientific Periodicals Eletronic Library SPELL (2003-2019). First, the conceptual origins, divergences and theoretical convergences are highlighted. Then, the evolution of scientific theoretical production is analyzed. Finally, an analysis of the themes and suggestions for future studies of the articles is carried out. It is concluded that there has been an increasing theoretical advance in the last two decades and that it may advance in the coming years. The contributions of the research agenda and understanding of how studies on the subject have been developed at the Brazilian national level stand out.
\end{abstract}

Keywords: Dynamic Capabilities. Theoretical evolution. Brazilian National Production. 


\section{INTRODUÇÃO}

O entendimento das Capacidades Dinâmicas (CDs) é procedente dos estudos em Administração Estratégica, especificamente em abordagens que utilizam a perspectiva da Visão Baseada em Recursos (RBV) (Kraaijenbrink, Spender \& Groen, 2010). Deste modo, os estudos das CDs buscam compreender como é possível sustentar a vantagem competitiva em situaçóes de mudanças ambientais (Teece, 2007).

A teoria das CDs destaca que as estratégias de uma organização se dão pelos recursos e competências desta (Sausen, 2012). Visto que, quanto mais rápido a concorrência agir de forma inovadora e dinâmica, mais determinante se torna o ambiente e a necessidade de ação e reação da organização para se manter sustentável e competitiva no mercado. Assim, a CDs das organizaçôes está diretamente ligada à busca de vantagens competitivas em um ambiente também competitivo (Martins \& Tavares, 2014).

Mesmo a CDs sendo um conceito bastante pesquisado na área de estratégia empresarial, os autores Hodgkinson e Healey (2011) evidenciam a falta de uniformidade ou de consenso em sua definição e dimensões. Di Stefano, Peteraf e Verona (2010) ressaltam que há uma disparidade em torno do construto desta temática, e o exercício de definição e compreensão teórica é importante para o avanço na área. O tema encontra-se em um estágio de variação e desenvolvimento de conceitos e modelos (Barreto, 2010).

Alguns estudos nacionais brasileiros buscaram por meio da bibliometria compreender a evolução da temática das CDs. O estudo de Bueno e Zarelli (2013) realizou uma análise bibliométrica nas bases Web of Science, Science Direct e Scopus sobre a relação das CDs enquanto fator de sucesso das pequenas e médias empresas. Picoli, Souza e Takahashi (2013) realizaram uma bibliometria dos artigos publicados sobre no período de 1997 a 2012.

Encontra-se ainda o estudo de Vicente et al. (2015), que realizaram uma análise bibliométrica dos artigos presentes nas bases originais de periódicos científicos em Administração e as publicaçóes em congressos de Administração, da Associação Nacional de Pós-Graduação e Pesquisa em Administração ANPAD. Já, Cardoso e Kato (2015) analisaram as publicaçóes sobre CDs entre 1992 e 2012 pelo site ISI Web of Knowledge. Ainda, Müller e Silveira-Martins (2016) realizaram um estudo bibliométrico em bases nacionais e internacionais (EBSCO, SPELL e ProQuest) sobre CDs no período de 1991-2014.

Mais recentemente, Kurek, Rocha e João (2018) buscaram por meio do estudo bibliométrico analisar a literatura acadêmica atual em capacidades de marketing na base de dados Web of Science no período de 1995-2018. E os autores, Miolo, Cappellari e Sausen (2018) efetuaram o levantamento dos artigos publicados na base SCOPUS no período de 2012 a 2017.

Neste viés, percebe-se um avanço acadêmico em compreender a evolução da temática por meio dos estudos bibliométricos e de análise da produção acadêmica. Ainda, elucida-se uma lacuna teórica sobre o desenvolvimento da produção acadêmica brasileira sobre CDs, visto que os estudos correlatos analisaram outros vieses da temática no estudo bibliométrico nacional e/ou internacional, desenvolvendo as análises com determinadas bases de dados e com critérios bibliométricos diferentes. Deste modo, ao analisar a produção científica nacional brasileira por meio de uma revisão sistemática da literatura, elucidando as tendências das produçóes, principais temáticas relacionadas e sugestôes de estudos futuros, destaca-se a relevância e originalidade deste estudo em relação a literatura já existente. 
Neste contexto, surge a pergunta norteadora deste estudo: Qual os principais debates teóricos sobre a origem e evolução da teoria das CDs, qual a evolução da temática e as principais proposiçóes para estudos futuros na produção científica brasileira? Deste modo, o objetivo deste estudo é debater a evolução da produção científica brasileira sobre a temática das Capacidades Dinâmicas por meio de uma revisão sistemática da literatura na base Scientific Periodicals Eletronic Library - SPELL, dentre os anos de 2000-2019.

Para realização do estudo, optou-se por adotar a Scientific Periodicals Eletronic Library - SPELL como base de dados. A SPELL é uma base de dados brasileira que concentra documentos publicados a partir de 2000, da área de Administração, Contabilidade e Turismo. Todos os documentos desta base de dados são open acess, o que representa um diferencial em relação às demais bases, especialmente às internacionais. Outro fator para o uso do SPELL foi em ter indexada a ela, revistas nacionais.

$\mathrm{Na}$ pesquisa realizada junto à base SPELL foi delimitado que o documento buscado tivesse o termo "Capacidades Dinâmicas" no título ou palavras-chave. A busca retornou 93 artigos, dos quais, um foi descartado por ser de publicação em periódico internacional. O recorte temporal realizado abrange as obras produzidas entre o ano 2000 e o mês de dezembro de 2019. A coleta dos dados na base foi efetivada no dia 16/12/2019.

Primeiramente efetuou-se a leitura dos resumos para analisar o enquadramento da temática das CDs nos artigos. Após, a identificação dos nomes dos periódicos, ano de publicação dos artigos foram localizadas nas informaçóes dos artigos na base SPELL. Os principais autores dos artigos analisados foram elucidados pelas informaçóes na identificação dos artigos e considerou-se apenas a autoria principal, descartando as coautorias, ou seja, analisou-se os autores que foram primeiros autores em mais de um artigo dentre os artigos localizados na base SPELL. Para elucidar as principais temáticas relacionadas a CDs nos artigos, analisou-se os resumos de cada artigo e categorizou-se conforme semelhança e aproximação teórica da temática relacionada. As sugestôes de estudos futuros indicados pelos artigos analisados foram sintetizadas por meio da leitura e interpretação das sessóes de conclusão dos estudos.

Primeiramente, busca-se apresentar um debate teórico sobre a evolução teórica sobre as CDs, destacando suas origens conceituais, divergências e convergências teóricas. Em seguimento, analisa-se a evolução da produção teórica científica nacional brasileira por meio dos principais periódicos e revistas científicas nacionais e pelos principais autores dos artigos analisados. Por fim, efetuou-se uma análise das temáticas e sugestôes de estudos futuros dos artigos.

\section{CAPACIDADES DINÂMICAS: ORIGENS E CONCEITOS}

A globalização e os crescentes avanços tecnológicos influenciam as organizações a buscar constantemente estratégias para sobreviver e prosperar em ambientes altamente dinâmicos e caracterizados por rápidas mudanças, tornando a administração uma função complexa e desafiadora. Diante disso, é necessário que as organizaçóes ponderem sobre os diferentes modelos de estratégia, buscando novas oportunidades de crescimento, considerando, também, o dinamismo e a incerteza do ambiente. Portanto, em um contexto como este, torna-se relevante o estudo das capacidades dinâmicas, haja visto que esta 
teoria busca explicar como as organizaçóes conseguem alcançar e sustentar vantagens competitivas ao longo do tempo (Teece, Pisano \& Shuen, 1997).

A vantagem competitiva pode ser entendida como a capacidade de as organizaçóes implantarem processos e recursos ainda não utilizados pelos concorrentes, gerando, assim, oportunidades e níveis de desempenho acima da média apresentada no mercado (Vasconcelos \& Cyrino, 2000). Recursos, estes, que podem ser de natureza tangível, intangível, humanos ou de todas as outras atividades executadas na organização (Adeniran, 2016).

Os estudos de vantagem competitiva iniciaram a partir da década de 70, sob a ótica de diferentes correntes do pensamento econômico e com uma disparidade de perspectivas teóricas. Algumas teorias anteriores destacam a obtenção de vantagens competitivas, como a abordagem da Organização Industrial (SPC), a abordagem da Visão Baseada em Recursos (RBV) e a abordagem dos Processos de Mercado, contudo, tais abordagens não consideram o dinamismo do ambiente e a forma como as organizaçóes operam nestes ambientes de forma mais enfática (Eisenhardt \& Martin, 2000). Assim, a teoria das capacidades dinâmicas é uma abordagem alternativa para explicar como as organizaçóes conseguem alcançar e manter vantagens competitivas em ambientes dinâmicos (Teece, Pisano \& Shuen, 1997).

A teoria das Capacidades Dinâmicas surge como uma nova abordagem da Administração Estratégica que, ao combinar o dinamismo do ambiente e os fatores internos da organização, é capaz de gerar vantagem competitiva sustentável (Teece, Pisano \& Shuen, 1997; Zahra \& George, 2002; Teece, 2007; Wang \& Ahmed, 2007). Buscando explicar como as organizaçóes agem para reconfigurar de forma proativa sua base de recursos, a perspectiva teórica das CDs defende a habilidade da organizaçáo integrar, construir e reconfigurar as competências externas e internas para adaptar-se rapidamente ao ambiente altamente mutável (Teece, Pisano \& Shuen, 1997; Sausen, 2012).

As CDs utilizam os pressupostos teóricos da RBV para explicar e compreender como as organizaçóes buscam reformular seus recursos organizacionais para adaptar-se as mudanças ocorridas no ambiente (Teece, Pisano \& Shuen, 1997). Diversos autores (Teece, Pisano, Schuen \& 1997; Eisenhardt \& Martin, 2000; Teece, 2007; Ambrosini \& Bowman, 2009; Ferreira et al., 2014) defendem que esta teoria surge para preencher a lacuna teórica deixada pela RBV, no sentido de tornar a vantagem competitiva sustentável em ambientes altamente dinâmicos e explicar como algumas organizaçôes respondem rapidamente às mudanças do ambiente externo.

A literatura aponta ainda que a origem e desenvolvimento teórico da teoria das CDs além de estar ligadas aos conceitos derivados da Teoria da Visão Baseada em Recursos (RBV) (Penrose, 1959; Wernerfelt, 1984; Barney, 1991), estão alinhadas à corrente de pensamento difundidas por Shumpeter (1942), que trazia um novo olhar sobre as ideias de inovação nas organizações, e também sobre as rotinas e competências oriundas da Teoria Comportamental da Firma (Cyert \& March, 1963). A RBV, em especial, tem sido utilizada como base para diversos estudos voltados para análise da vantagem competitiva empresarial. A origem dessa abordagem competitiva é baseada em um grande número de ensaios teóricos, começando com a obra "The Nature of the Firm" de Coase (1937), e a principal vertente teórica da RBV é oriunda do trabalho seminal de Edith Penrose de 1959, onde analisa a firma a partir de um conjunto de recursos (Penrose, 1959). A transferência conceitual da RBV para a literatura da administração estratégica é geralmente atribuída a Wernefelt (1984), sendo, subsequentemente, difundida por Barney (1991). 
Na década de 90, retomando a análise interna das organizaçóes, a RBV passou a ser difundida com maior amplitude. Essa teoria destaca o desempenho superior baseado nas diferenças do ambiente interno das empresas. Considerando essa lógica, as organizaçóes podem ser consideradas como um conjunto de recursos tangíveis e intangíveis, logo, a diferença entre as organizaçôes advém da experiência baseada na trajetória e dos ativos, habilidades e da cultura organizacional adquiridas. Deste modo, a vantagem competitiva se desenvolvia a partir de recursos valiosos e raros de serem imitáveis por outras organizaçóes, assim como a essência da estratégia em organizar recursos e capacidades para alavancar a organizaçáo (Barney, 1991).

Os primeiros estudos sobre as capacidades dinâmicas foram iniciados por Winter (1964), contudo, este tema passou a ter maior relevância a partir da década de 90, com os estudos de Teece, Pisano e Shuen (1997) e, em seguida, Eisenhardt e Martin (2000), visto o aumento das pesquisas no âmbito estratégico em virtude da redução das fronteiras entre os mercados e o consequente aumento da concorrência entre as organizaçôes (Martins \& Tavares, 2014). Desde a sua primeira abordagem, esta temática vem ganhando destaque em pesquisas no campo da Administração, atraindo atenção como um paradigma emergente (Barreto, 2010) em pesquisas internacionais (Ambrosini \& Bowman, 2009; Barreto, 2010) e nacionais em função de requerer investigação profunda e sistemática da sua complexidade (Guerra, Tondolo \& Camargo, 2016).

\section{DIVERGÊNCIAS E CONVERGÊNCIAS TEÓRICAS}

Além das várias definiçôes encontradas na literatura para o termo CDs, diversas também são as designaçóes quanto aos seus constructos (ou elementos componentes destas capacidades em uma organização). Segundo estudo de Meirelles e Camargo (2014), parte dos autores enfatizam as CDs como sendo um conjunto de processos e rotinas organizacionais, caso de Teece, Pisano e Shuen (1997), Eisenhardt e Martin (2000) e Zollo e Winter (2002), enquanto outro grupo de autores refere como sendo habilidades, comportamentos e capacidades organizacionais, como Collis (1994), Andreeva e Chaika (2006), Helfat et al. (2007) e Wang e Ahmed (2007).

Com relação ao primeiro grupo, pode-se citar os entendimentos de Teece, Pisano e Shuen (1997), para o qual as CDs são operacionalizadas na forma de fatores compostos por processos, posiçóes e trajetórias; Eisenhardt e Martin (2000), que mencionam como componentes das CDs os processos ou rotinas organizacionais e as estratégias pelas quais a organização alcança novas configuraçóes de recursos; ou ainda Zollo e Winter (2002), que atribuem os processos de acumulação de experiências, processos de articulação e codificação do conhecimento e, ainda, o ciclo recursivo de aprendizagem como tais componentes.

Dentre os autores que enfatizam as CDs como conjunto de capacidades, habilidades e comportamentos está Collis (1994). O autor menciona as capacidades operacionais, as habilidades em desenvolver novas estratégias rapidamente (ou melhor do que a concorrência) e a capacidade de aprender a aprender como elementos componentes. Destaca, portanto, à velocidade de operação perante a concorrência. Da mesma forma, Helfat et al. (2007) sugerem a habilidade para alterar a base de recursos, a capacidade de atuar de forma recorrente para modificar esta base e a capacidade de buscar e selecionar 
aquelas modificaçóes relevantes como elementos componentes das CDs, ressaltando o poder de deliberação da empresa.

Conforme afirmam Guerra, Tondolo e Camargo (2016) as CDs podem ser fonte de competitividade na medida em que os processos de mudança da base de recursos e de capacidades organizacionais sejam intencionais, ou seja, dirigidos pela gestão das organizaçóes face às exigências competitivas do mercado. Esta abordagem também podem ser fonte de vantagem competitiva na medida em que os concorrentes ficam impossibilitados de copiar ou duplicar um valor criado, contribuindo tanto para o alcance quanto para a manutenção da competitividade ao longo do tempo (Ambrosini \& Bowman, 2009; Danneels, 2010; Helfat et al., 2007; Zahra, Sapienza \& Davidsson, 2006).

\section{ANÁLISE DA EVOLUÇÃO DAS PUBLICAÇÕES BRASILEIRAS}

Buscando analisar a evolução das publicaçóes ao longo dos anos, apresenta-se na Figura 1 o número de artigos publicados nos periódicos no período de 2003-2019. Observa-se que o ponto de partida das publicaçóes sobre CDs na base SPELL iniciou-se no ano de 2003.

Figura 1. Evoluçáo das publicaçôes por ano

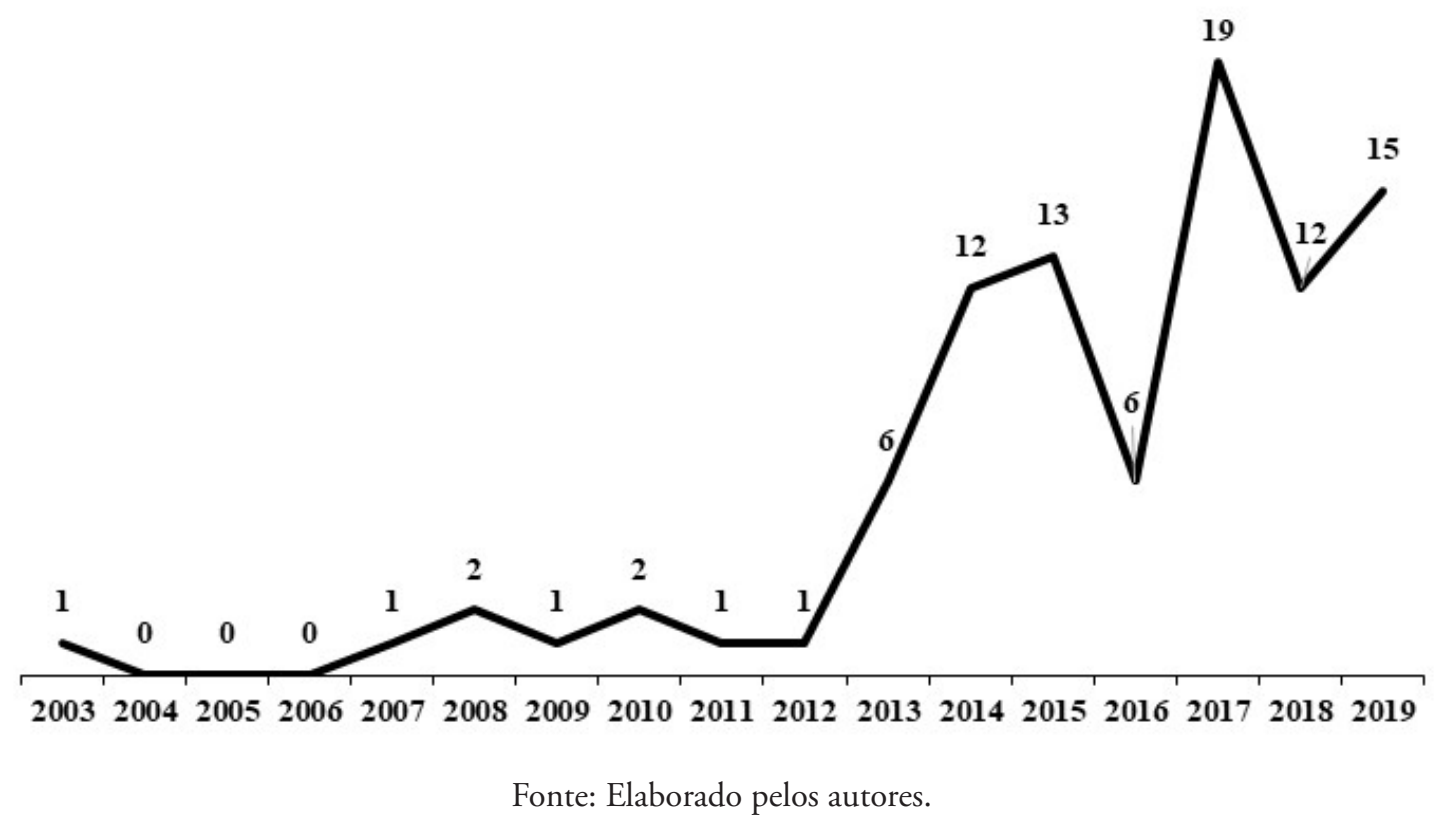

Em análise da Figura 1, percebe-se que o início das publicaçóes sobre CDs foi no ano de 2003. Do ano de 2002 até 2006 não houveram publicaçôes sobre a temática em periódicos pertencentes a base. Do ano de 2007 até o ano de 2012 houveram 8 artigos publicados. Destaca-se que a partir do ano de 2012 até o ano de 2015 houve um aumento considerável de publicaçóes sobre a temática, tendo no período 2012-2015 um total de 26 artigos publicados.

No ano de 2015 até 2016 houve uma pequena queda na quantidade de artigos publicados, tendo no ano de 2016 um total de 6 artigo publicados sobre a temática. No ano seguinte, 2017, houve um aumento da quantidade de artigos publicados, alcançando o número de 19 artigos publicados neste ano. $\mathrm{O}$ ano de 2018 teve um total de 12 artigos publicados, seguidos de 15 artigos publicados sobre a 
temática no ano de 2019. Percebe-se assim, que nos anos finais do período considerado (2003-2019) há um aumento da publicação dos estudos, demonstrando que a temática das CDs vem sendo estudada por um número cada vez maior de pesquisadores no âmbito dos periódicos nacionais.

Inicialmente são analisados os periódicos nacionais dos respectivos artigos localizados na base SPELL. A Tabela 1, apresenta o número de artigos por periódico e o percentual do total de artigos localizados.

Tabela 1. Número de artigos publicados por periódicos

\begin{tabular}{|c|c|c|}
\hline Periódicos Nacionais & $\begin{array}{c}\text { Artigos } \\
\text { Publicados }\end{array}$ & $\%$ \\
\hline Revista Ibero-Americana de Estratégia & 10 & 10,9 \\
\hline Revista de Administração de Empresas & 7 & 7,6 \\
\hline Revista de Administração e Inovação & 6 & 6,5 \\
\hline Revista de Administração Contemporânea & 5 & 5,4 \\
\hline InternexT & 4 & 4,3 \\
\hline Brazilian Business Review & 4 & 4,3 \\
\hline Revista Alcance & 4 & 4,3 \\
\hline $\begin{array}{l}\text { Revista de Empreendedorismo e Gestão de } \\
\text { Pequenas Empresas }\end{array}$ & 3 & 3,3 \\
\hline Gestão e Planejamento & 2 & 2,2 \\
\hline NAVUS - Revista de Gestão e Tecnologia & 2 & 2,2 \\
\hline Perspectivas em Gestâo \& Conhecimento & 2 & 2,2 \\
\hline PODIUM: Sport, Leisure and Tourism Review & 2 & 2,2 \\
\hline Revista ADM.MADE & 2 & 2,2 \\
\hline Revista Brasileira de Gestão de Negócios & 2 & 2,2 \\
\hline Revista Ciências Administrativas & 2 & 2,2 \\
\hline Revista de Administração da UFSM & 2 & 2,2 \\
\hline Revista de Administração Mackenzie & 2 & 2,2 \\
\hline Revista Eletrônica Científica do CRA-PR & 2 & 2,2 \\
\hline Revista Eletrônica de Estratégia e Negócios & 2 & 2,2 \\
\hline Revista Gestão \& Tecnologia & 2 & 2,2 \\
\hline $\begin{array}{l}\text { Caderno Profissional de Administração da } \\
\text { UNIMEP }\end{array}$ & 1 & 1,1 \\
\hline Cadernos EBAPE.BR & 1 & 1,1 \\
\hline Desafio Online & 1 & 1,1 \\
\hline FACES: Revista de Administração & 1 & 1,1 \\
\hline Gestão \& Regionalidade & 1 & 1,1 \\
\hline Gestão e Conexões & 1 & 1,1 \\
\hline Gestão e Sociedade & 1 & 1,1 \\
\hline Organizações \& Sociedade & 1 & 1,1 \\
\hline $\begin{array}{l}\text { RACE: Revista de Administração, Contabilidade e } \\
\text { Economia }\end{array}$ & 1 & 1,1 \\
\hline
\end{tabular}




\begin{tabular}{|c|c|c|}
\hline RAUSP - Revista de Administração & 1 & 1,1 \\
\hline REGE Revista de Gestão & 1 & 1,1 \\
\hline Reuna & 1 & 1,1 \\
\hline Revista Brasileira de Estratégia & 1 & 1,1 \\
\hline Revista Brasileira de Inovação & 1 & 1,1 \\
\hline Revista Capital Científico - Eletrônica & 1 & 1,1 \\
\hline Revista de Administração da Unimep & 1 & 1,1 \\
\hline Revista de Administração Pública & 1 & 1,1 \\
\hline Revista de Ciências da Administração & 1 & 1,1 \\
\hline Revista de Negócios & 1 & 1,1 \\
\hline Revista Gestão Organizacional & 1 & 1,1 \\
\hline $\begin{array}{l}\text { Revista Pensamento Contemporâneo em } \\
\text { Administração }\end{array}$ & 1 & 1,1 \\
\hline Sociedade, Contabilidade e Gestão & 1 & 1,1 \\
\hline Tecnologias de Administração e Contabilidade & 1 & 1,1 \\
\hline Turismo em Análise & 1 & 1,1 \\
\hline Turismo: Visão e Ação & 1 & 1,1 \\
\hline Total por Ano & 92 & 100,0 \\
\hline
\end{tabular}

Fonte: Elaborado pelos autores.

Percebe-se na Tabela 1 os diferentes periódicos nacionais que publicaram artigos sobre a temática das CDs no período de 2003-2019. Destaca-se que o periódico que mais teve publicaçóes sobre a temática considerada foi a Revista Ibero-Americana de Estratégia com 10 artigos publicados. Em seguimento, a Revista de Administração de Empresas teve 7 artigos publicados, a Revista de Administração e Inovação teve um total de 6 artigos publicados e a Revista de Administração Contemporânea teve 5 artigos publicados sobre a temática das Capacidades Dinâmicas. Outros periódicos também tiveram publicaçóes consideradas, contudo com um número de artigos menor que as destacadas.

Buscando identificar quais os principais autores sobre a temática, analisou-se quais os autores dentre os artigos localizados que tiveram mais de uma publicação de artigos. Para isso, considerou-se apenas a autoria principal, descartando as coautorias, ou seja, analisou-se os autores que foram primeiros autores em mais de um artigo dentre os artigos localizados na base SPELL. A Tabela 2 apresenta a quantidade de artigos publicados por autor.

Tabela 2. Quantidade de artigos publicados por autor

\begin{tabular}{cc}
\hline AUTOR & ARTIGOS \\
CARDOSO, A. L. J. & 3 \\
\hline CRISTIANE FROEHLICH, C. & 3 \\
\hline TONDOLO, V. A. G. & 3 \\
\hline
\end{tabular}

Fonte: Elaborado pelos autores. 
Conforme os dados apresentados na Tabela 2, destaca-se os principais pesquisadores que tiveram três publicaçôes como autores principais no período de 2003-2019 na base SPEL: a) Dr. André Luís Janzkovski Cardoso, professor da Universidade Federal de Mato Grosso - UFMT; b) Dra ${ }^{\mathrm{a}}$. Cristiane Froehlich, professora da Universidade Feevale - FEEVALE e; d) Dr. Vilmar Antônio Gonçalves Tondolo, professor da Universidade Federal de Pelotas - UFPEL.

\section{ANÁLISE DAS TEMÁTICAS E SUGESTÕES DE PESQUISAS FUTURAS DOS ARTIGOS}

Para elucidar as principais temáticas relacionadas a capacidade dinâmica nos artigos analisados, analisou-se os resumos de cada artigo e categorizou-se conforme semelhança e aproximação teórica da temática relacionada. A Tabela 3 apresenta as principais temáticas relacionadas aos CDs nos artigos analisados.

Tabela 3. Principais temáticas dos artigos

\begin{tabular}{lcc}
\hline \multicolumn{1}{c}{ TEMÁTICA } & ARTIGOS & $\%$ \\
\hline Inovação & 12 & 13,04 \\
\hline Evolução Conceitual e Teórica & 10 & 10,87 \\
\hline $\begin{array}{l}\text { Desenvolvimento das Capacidades } \\
\text { Dinâmicas }\end{array}$ & 8 & 8,70 \\
\hline Tecnologias & 7 & 7,61 \\
\hline Internacionalização & 6 & 6,52 \\
\hline Rotinas e Processos Organizacionais & 5 & 5,43 \\
\hline Sustentabilidade & 5 & 5,43 \\
\hline Aprendizagem & 4 & 4,35 \\
\hline Desempenho & 3 & 3,26 \\
\hline Empreendedorismo & 3 & 3,26 \\
\hline Marketing & 3 & 3,26 \\
\hline RBV & 3 & 3,26 \\
\hline Sistemas de Gestão & 3 & 3,26 \\
\hline Conhecimento & 2 & 2,17 \\
\hline Modelo de Negócio & 2 & 2,17 \\
\hline Terceirização de Serviços & 2 & 2,17 \\
\hline Outros & 14 & 15,22 \\
\hline & $\mathbf{9 2}$ & $\mathbf{1 0 0}$ \\
\hline
\end{tabular}

Fonte: Elaborado pelos autores.

Neste contexto, destaca-se que a Inovação é uma das temáticas mais relacionadas as CDs, aparecendo em 12 artigos (13,04\%) dos artigos analisados. Outra temática relacionada refere-se a sua evolução conceitual e teórica, aparecendo em 10 artigos (10,87\%). O processo de desenvolvimento das CDs é relacionado em 8 artigos (8,70\%) e o uso de tecnologias aparece em 7 artigos (7,61\%) dos artigos analisados. 
O processo de internacionalização de empresas é outra temática relacionada à CDs, tendo 6 artigos $(6,52 \%)$ abordado essa relação. Os processos e rotinas organizacionais e a sustentabilidade também foram relacionados em 5 artigos $(5,43 \%)$ respectivamente. A aprendizagem organizacional foi relacionada em 4 artigos (4,35\%) sobre CDs.

As temáticas de aprendizagem, desempenho, empreendedorismo, marketing, RBV e sistemas de gestão foram relacionadas as CDs em 3 artigos (3,26\%) respectivamente. Já, as temáticas de conhecimento, modelo de negócio e terceirização de serviços foram analisadas em 2 artigos (2,17\%) respectivamente. Ainda, destaca-se que 14 artigos $(15,22 \%)$ relacionaram as CDs a outras temáticas.

Por fim, buscando analisar as sugestôes de estudos futuros indicados pelos artigos analisados, por meio da leitura e interpretaçáo das sessóes de conclusão dos estudos, categorizou-se as sugestóes de estudos futuros. A Tabela 4 apresenta a síntese das sugestóes de estudos futuros

Tabela 4. Síntese das sugestôes de estudos futuros indicados pelos artigos

\section{SÍNTESE DAS SUGESTÓES DE ESTUDOS FUTUROS}

Analisar a capacidade de inovação sustentável em indústrias

Analisar a influência da ambidestria organizacional no desenvolvimento de Capacidades Dinâmicas

Analisar as correlaçóes existentes entre as tecnologias Big Data, IoT e Big Data com as Capacidades Dinâmicas

Analisar os elementos componentes das Capacidades Dinâmicas

Análise de co-citação em diferentes periódicos sobre a teoria das estratégias e Capacidades Dinâmicas

Análises quantitativas sobre o desenvolvimento de vantagem competitiva sustentável com Capacidades

Dinâmicas

Análises quantitativas sobre o processo de internacionalização e Capacidades Dinâmicas

Aprofundamento teórico sobre os aspectos que tangenciam a abordagem processual das Capacidades Dinâmicas

Compreender a influência das Capacidades Dinâmicas para a inovação no nível estratégico

Compreender o desenvolvimento da capacidade absortiva em estudos longitudinais

Compreender os micro fundamentos da capacidade inovativa

Desenvolver ferramentas metodológicas para mensurar Capacidades Dinâmicas e inovação em serviços

envolvidas na implementação e manutençâo de práticas de sustentabilidade empresarial

Diferenças entre os campos teórico e prático sobre Gestão Sustentável da Cadeia de Suprimentos e

desenvolvimento de Capacidades Dinâmicas

Entender o desenvolvimento de Capacidades Dinâmicas em organizações hibridas

Estudo de casos múltiplos no desenvolvimento de Capacidades Dinâmicas

Estudos quantitativos da relação da internacionalização e Capacidades Dinâmicas

Estudos quantitativos sobre a relação entre aprendizagem organizacional e capacidades tecnológicas

Evidenciar a operacionalização dos processos e as açóes específicas das redes de inovação e Capacidades

Dinâmicas

Investigar as analisar as Capacidades Dinâmicas como um antecedente dos recursos intangíveis

Medir o efeito do constructo das Capacidades Dinâmicas no terceiro setor na captação de recursos, voluntários, parceiros e apoiadores

Modelos quantitativos da relação de gestão sustentável e Capacidades Dinâmicas

Novos modelos de mensuração das Capacidades Dinâmicas associados à inovação

Propor modelos para mensurar a capacidade de transferência tecnológica 
Relacionar as Capacidades Dinâmicas com o Sistema de Qualidade ou de Gestão de Resíduos

Uso de modelagem de equaçóes estruturais na análise da relação da Orientação empreendedora e ao mercado com Capacidades Dinâmicas

Fonte: Elaborado pelos autores.

As análises da Tabela 4 permitem inferir algumas possibilidades de estudos futuros integrando as CDs e outras temáticas. Percebe-se que os estudos analisados apontaram lacunas que ainda permeiam o desenvolvimento das CDs no âmbito nacional, suas sugestôes vão ao encontro com a continuidade dos estudos efetuados e agregam outras possibilidades de análises e relaçôes necessárias para os avanços dos estudos das Capacidades Dinâmicas.

Percebe-se que o estudo acerca das estratégias organizacionais se torna relevante em virtude do atual contexto do mercado, marcado por forte concorrência, contínuas mudanças e instabilidades. Esta temática vem ganhando considerável importância nos últimos anos, pois busca compreender a forma como as organizaçôes desenvolvem, sustentam e como utilizam seus recursos, visando a obtenção de vantagem competitiva.

Permeado de sobreposiçóes conceituais, semelhanças de entendimento e lacunas (Guerra, Tondolo \& Camargo, 2016) a literatura sobre capacidades dinâmicas apresenta fortes controvérsias sobre os antecedentes e elementos componentes de desenvolvimento (Meirelles \& Camargo, 2014). Corroborando o exposto, Guerra, Tondolo e Camargo (2016) argumentam que uma das lacunas existentes na literatura de capacidades dinâmicas diz respeito ao seu processo de desenvolvimento, função da origem e efeito dos recursos que as geram. Portanto, se faz necessário estudos que possam desvendar o modo como as organizaçóes desenvolvem capacidades dinâmicas.

\section{CONCLUSÕES}

Conclui-se que evolução das publicaçóes da temática iniciou-se no ano de 2003. No período analisado (2003-2019), o maior volume de publicaçóes efetuou-se nos últimos 6 anos da análise (20142019). Neste contexto, percebe-se o crescimento das publicaçóes nacionais de artigos que buscam compreender e analisar as CDs e destaca os esforços em contribuir com o avanço da temática. Permite-se ponderar que, houve um crescente interesse acadêmico pelo tema nas duas últimas décadas.

Os resultados apontam que os periódicos que mais centralizaram as publicaçôes de artigos sobre a temática foram a Revista Ibero-Americana de Estratégia, Revista de Administração de Empresas, Revista de Administração e Inovação e a Revista de Administração Contemporânea. O estudo aponta ainda os principais autores dos artigos analisados conforme a autoria principal, destacando o empenho dos pesquisadores nacionais no desenvolvimento da temática.

Dentre as temáticas relacionadas as CDs, conclui-se que a inovação é a mais utilizada nos artigos. $\mathrm{Na}$ análise das sugestóes de estudos futuros dos artigos, diversas proposiçóes foram apresentadas, o que possibilita a continuidade e avanço da temática no âmbito nacional. Contudo, percebeu-se que a maioria dos estudos apenas sugere a replicação do próprio estudo em outra amostra ou segmento. Ainda, muitos estudos não apresentam a sugestão de estudos futuros. 
Desde o conceito inicial desenvolvido por Teece, Pisano e Shuen (1997), vêm surgindo um número considerável e crescente de pesquisas acadêmicas focadas nas CDs. Nos últimos três anos, há relaçóes dos conceitos em diversas áreas da gestão, como, marketing, empreendedorismo, gerenciamento estratégico, etc. Percebe-se, devido aos esforços teóricos no sentido de desenvolver e expandir o conceito no cenário acadêmico, o desenvolvimento teórico das CDs cresceu muito desde a sua concepçáo teórica original proposta pelos autores. Pondera-se que a abordagem das CDs pode possuir muitos significados dependendo da corrente teórica dos autores, porém identificam-se, na maioria das definiçóes, elementos convergentes, semelhantes e até algumas controvérsias sobre os elementos e componentes que constituem as CDs.

Como limitaçóes do estudo pode-se citar que, mesmo que a base de dados adotada na pesquisa cumpriu com os objetivos do estudo, a inclusão de outras bases de dados nacionais e internacionais poderiam qualificar as análises bibliométricas realizadas. Sugere-se que estudos futuros adotem as relaçóes e proposiçóes aqui debatidas para fundamentar e avançar nos estudos e análises teóricas/empíricas sobre a teoria das CDS, com destaque em seu processo de desenvolvimento nas organizaçóes.

\section{REFERÊNCIAS}

Adeniran, T. V., \& Johnston, K. A. (2016). The impacts of ICT utilisation and dynamic capabilities on the competitive advantage of South African SMEs. Journal of Information Technology and Management, v. 5 , n. 1 , p. 59-89.

Ambrosini, V., \& Bowman, C. (2009). What are dynamic capabilities and are they a useful construct in strategic management? International Journal of Management Reviews, 11(1), 29-49.

Andreeva, T., \& Chaika, V. (2006). Dynamic Capabilities: what they need to be dynamic? St. Petersburg State University. São Petersburgo.

Barney, J. B. (1991). Firms resources and sustained competitive advantage. Journal of Management, v. 17, n. 1 .

Barreto, I. (2010). Dynamic capabilities: A review of past research and an agenda for the future, Journal of Management, 36(1), 256-280.

Bueno, E., \& Zarelli, P. R. (2013). Capacidades dinâmicas como fator de sucesso em pequenas e médias empresas - PMEs: uma análise bibliométrica, In: III Congresso Internacional do Conhecimento e Inovação. Anais... Porto Alegre.

Cardoso, A. L. J., \& Kato, H. T. (2015). Análise das publicaçôes sobre capacidades dinâmicas entre 1992 e 2012: discussóes sobre a evolução conceitual e as contribuiçóes dos autores de maior notoriedade na área, RAM, Rev. Adm. Mackenzie, 16(3), 201-237.

Collis, D. J. (1994). Research note: how valuable are organizational capabilities? Strategic Management Journal, 15, 143-152. 
Cyert, R., \& March, J. (1963). A behavioral theory of the firm. Englewood Cliffs: Prentice-Hall.

Danneels, E. (2010). Trying to become a different type of company; dynamic capability at Smith Corona, Strategic Management Journal, 32(1), 235-264.

Di Stefano, G., Peteraf, M. A., \& Verona, G. (2010). Dynamic capabilities deconstructed: A bibliographic investigation into the origins, development and future directions of the research domain, Industrial and Corporate Change, 19(4), 1187-1204.

Eisenhardt, K. M., \& Martin, J. A. (2000). Dynamic Capabilities: What are they? Strategic Management Journal, 21(10-11), 1105-1121.

Ferreira, M. A. S. P. V., Reis, N. R., Serra, F. A. R., \& Costa, B. K. (2014). Entendendo a pegada da VBR nos estudos em negócios internacionais: os últimos vinte anos de pesquisa. Brazilian Business Review, v.11, n. 4, p. 57-90.

Guerra, R. M. A., Tondolo, V. A. G., \& Camargo, M. E. (2016). O que (ainda) podemos aprender sobre capacidades dinâmicas, Revista Ibero-Americana de Estratégia, 15(1), 44-64.

Helfat, C., Finkelstein, S., Mitchell, W., Peteraf, M., Singh, H., Teece, D., \& Winter, S. (2007). Dynamic Capabilities: Understanding Strategic Changes In Organizations. Malden: Blackwell Publishing.

Hodgkinson, G. P., \& Healey, M. (2011). Psychological foundations of dynamic capabilities: Reflexion and reflection in strategic management, Strategic Management Journal. Strategic Management Journal, 32(13), 1500-1516.

Kraaijenbrink, J., Spender, J.-C., \& Groen, A. J. (2010). The resource based view: a review and assessment of its critiques, Journal of Management, 36(1), 349-372.

Kurek, P., Rocha, T. V., \& João, B. N. (2018). Um mapeamento sistemático na pesquisa em capacidades de marketing, In: $13^{\circ}$ Congresso Latino-Americano de Varejo e Consumo. Anais... Rio de Janeiro.

Martins, S. E., \& Tavares, P. M. (2014). Processo de formulação de estratégias: capacidade mercadológica, incerteza ambiental e desempenho, Organizaçôes em contexto, 10(20), 297-322.

Meirelles, D. S., \& Camargo, A. A. B. (2014). Capacidades Dinâmicas: O Que São e Como Identificálas? Revista de Administração Contemporânea, 18, 41-64.

Miolo, M. A., Cappellari, G., \& Sausen, J. O. (2018). Um estudo bibliométrico em base de dados internacionais, In: Saláo do Conhecimento 2018. Anais... Ijuí.

Müller, C. R., \& Silveira-Martins, E. (2016). Capacidades dinâmicas: um estudo bibliométrico em bases nacionais e internacionais, In: XXIX Congreso Latinoamericano de Estrategia. Anais...

Penrose, E. (1959). The theory of growth of the firm. New York: Wiley. 
Picoli, F. R., Souza, C. P. S., \& Takahashi, A. R. W. (2013). Produção Nacional em Capacidades Dinâmicas: Um Estudo Bibliométrico, In: XXXVII Encontro da Anpad. Anais... Rio de Janeiro.

Sausen, J. O. (2012). Gestão estratégica, competitividade e desenvolvimento. In: Siedenberger, Dieter (Org.). Desenvolvimento sob múltiplos olhares. Ijuí: Unijuí, 207-267.

Teece, D. J. (2007). Explicating dynamic capabilities: the nature and microfoundations of (sustainable) enterprise performance, Strategic Management J, 28(13), 1319-1350.

Teece, D. J., Pisano, G., \& Shuen, A. (1997). Dynamic capabilities and strategic management, Strategic Management Journal, 18(7), 509-533.

Vasconcelos, F. C., \& Cyrino, Á. B. (2000). Vantagem Competitiva: os Modelos Teóricos Atuais e a Convergência Entre Estratégia e Teoria Organizacional. Revista de Administração de Empresas, v. 40, n. 4, p. 20-37.

Vicente, A. R. P., Rosa, A. R., \& Takahashi, A. R. W. (2015). Capacidades dinâmicas: um estudo bibliométrico a partir de publicaçôes em periódicos e eventos científicos nacionais, In: XVIII SEMEAD Seminários em Administração. Anais...

Wang, C. L., \& Ahmed. P. K. (2007). Dynamic capabilities: A review and research, International Journal of Management Reviews, 9, 31-51.

Wernerfelt, B. (1984). A resource-based view of the firm. Strategic Management Journal, v. 5.

Winter, S. G. (1964). Economic 'Natural Selection' and the Theory of the Firm, Yale Economic Essays, 4, $225-272$.

Zahra, S. A., \& George, G. (2002). Absorptive capacity: a review, reconceptualization, and extension, Academy of Management Review, 27(2), 185-203.

Zahra, S. A., Sapienza, H. J., \& Davidsson, P. (2006). Entrepreneurship and dynamic capabilities: a review, model and research agenda, Journal of Management Studies, 43(4), 917-955.

Zollo, M., \& Winter, S. G. (2002). Deliberate Learning and the Evolution of Dynamic Capabilities, Organization Science. 13(3), 339-351. 